\title{
Spatial scale of geomagnetic Pc5/Pi3 pulsations as a factor of their efficiency in generation of geomagnetically induced currents
}

\author{
Nadezda V Yagova ${ }^{1,2^{*}}$, Vyacheslav A Pilipenko ${ }^{1,2}$, Yaroslav A Sakharov ${ }^{3}$ and Vasily N Selivanov ${ }^{4}$
}

\footnotetext{
${ }^{*}$ Correspondence: nyagova@ifz.ru

${ }^{1}$ Schmidt Institute of Physics of

the Earth of the Russian Academy

of Sciences (IPE RAS), B.

Gruzinskaya ul, 10, Moscow,

Russia

Full list of author information is

available at the end of the article
}

\begin{abstract}
Geomagnetically induced currents (GICs) in a meridional power transmission line at the Kola Peninsula are analyzed during the intervals of Pc5/Pi3 (frequency range from 1.5 to $5 \mathrm{mHz}$ ) pulsation activity observed at the IMAGE magnetometer network. We have analyzed GIC in a transformer at the terminal station Vykhodnoj $\left(68^{\circ} \mathrm{N}, 33^{\circ} \mathrm{E}\right)$ during the entire year of 2015 , near the maximum of 24-th Solar cycle. To quantify the efficiency of GIC generation by a geomagnetic pulsation, a ratio between power spectral densities of GIC and magnetic field variations is introduced. Upon examination of the efficiency of geomagnetic pulsations in GIC generation, the emphasis is given to its dependence on frequency and spatial scale. To estimate pulsation spatial scales in latitudinal and longitudinal directions, the triangle of stations KEV-SOD-KIL has been used. Large-scale pulsations along the electric power line (with a high spectral coherence, low phase difference, and similar amplitudes) are found to be more effective in $\mathrm{GIC}$ generation than small-scale pulsations. The accuracy of GIC prediction also depends on the pulsation scale transversal to the electric power line.
\end{abstract}

Keywords: geomagnetically-induced currents; Pc5/Pi3 geomagnetic pulsations

\section{Introduction}

Space weather perturbations caused by the interaction of solar ejecta with the near-Earth environment activate global electromagnetic and plasma processes: intensification of the magnetosphere - ionosphere current systems, energization of ring current and radiation belt particles, enhanced precipitation into the auroral oval, disturbance of the geomagnetic field, etc. These processes are potential risky for space and ground technologies. Generation of geomagnetically induced currents (GICs) related to abrupt changes of the geomagnetic field is one of the most significant space weather factors for power transmission lines (e.g., Boteler (2001); Kappenman (2004)). Therefore, the geophysical community is making tremendous efforts to provide a global computer model of storm/substorm activity augmented by the magnetotelluric reconstruction of telluric currents (Love et al, 2018; Pulkkinen, 2015; Pulkkinen et al, 2007).

However, the highest risk of GIC may be related not only to global processes with enormous energy yield (e.g., for typical substorm it is of $10^{14} \mathrm{~J}$ ), but also to more localized and rapid processes. The interaction between the solar wind and the magnetosphere results in the occurrence of diverse types of nonstationary perturbations 
with various spatial and time scales. Such localized and fast disturbances embedded into the global magnetospheric processes may be the actual drivers of GIC bursts (Belakhovsky et al, 2019). In general, the amplitudes of geomagnetic variations decrease with frequency, whereas the induced electric field magnitudes are expected to grow with frequency. Therefore, the GIC response, which is a convolution of both factors, must have a maximum at some frequencies. Many case studies of GIC bursts demonstrated that this characteristic time scale is about $2-10 \mathrm{~min}$, that is, it falls into the frequency range of $\mathrm{Pc} 5 / \mathrm{Pi} 3$ pulsations, i.e. at the low-frequency flank of the ultra-low frequency (ULF) range.

The impulsive geomagnetic disturbances, that appear in ground-based magnetometer records during nighttime, may be associated with substorm onsets and subsequent activations, magnetic perturbation events - isolated disturbance of the geomagnetic field with the duration of about 10 minutes (Engebretson et al, 2019), intense geomagnetic pulsations Ps6/Pi3 - quasi-periodic series of impulses with duration 10-20 min, and narrow band Pc5 pulsations $2-7 \mathrm{mHz}$ ). Though the power of such processes is lower than the power of magnetospheric storms and substorms, the rapidly varying electromagnetic fields of these events can induce a significant GIC (Apatenkov et al, 2004; Belakhovsky et al, 2018; Viljanen, 1998; Yagova et al, 2018). Apatenkov et al (2020); Belakhovsky et al (2019) presented cases, where the ULF variations of the Pi3 or Ps6 type induced GIC in power lines up to $120 \mathrm{~A}$.

Viljanen et al (2001) suggested that the Pc5 waves during the recovery phase of a magnetic storm may cause intense GICs. Pulkkinen and Kataoka (2006) further suggested that a moderate and steady wave activity could lead to cumulative GIC effects such as corrosion of natural gas pipelines Especially global Pc5 waves could be very effective GIC drivers. The actual driver of GICs - the telluric electric field, can be estimated for a given magnetic field $B(f)$ varying with frequency $f$ above a homogeneous ground with conductivity from the boundary impedance condition (in the plane wave approximation $E / B=\omega^{1 / 2}(\mu \sigma)^{-1 / 2}$, where $\left.\omega=2 \pi f\right)$. For the Pc5 pulsations with $\omega=0.01 \mathrm{~s}^{-1}$ and the average conductivity in Fennoscandia $\sigma=$ $10^{-4} \mathrm{~S} / \mathrm{m}^{2}$ this relation gives $\mathrm{E}[\mathrm{mv} / \mathrm{km}] / \mathrm{B}[\mathrm{nT}] \approx 12 \mathrm{mV} / \mathrm{km} / \mathrm{nT}$. For the global Pc5 pulsations with $B \approx 100 \mathrm{nT}$, the expected telluric field can reach $E \approx 10^{3} \mathrm{mV} / \mathrm{km}$. This is almost as high as the estimate given by (Lucas et al., 2020) for the extreme once-per-century electrotellutic field over the US territory.

However, in all the previous studies of the relationship between GIC and geomagnetic variations, it was implicitly assumed that the magnetic field is homogeneous along the electric power line (EPL). The role of a geomagnetic variation spatial scale has never been thoroughly examined, although the importance of this effect has been postulated (e..g. Boteler and Pirjola (2017); Yagova et al (2018). Recently, it was indicated that the large-scale ULF pulsations may be more efficient in the GIC excitation Sakharov et al (2020). This result prompts that the ULF pulsation spatial scale may be an important though an underestimated factor in GIC generation. Here, we consider in detail the GIC dependence on pulsation spatial scale.

\section{Data Set and Event Analysis Technique}

The data of the IMAGE magnetometer network (10-sec cadence) (Tanskanen, 2009) is used for the analysis of the Pc5 pulsations. The GIC data is taken from the GIC 
system of monitoring on electric power lines (EPL). The system was deployed in the Kola Peninsula by the Polar Geophysical Institute and the Center for Northern Energetics (Viljanen et al, 2012). We use the data from the terminal station Vykhodnoi (VKH) at the $330 \mathrm{kV}$ power line located at the corrected geomagnetic (CGM) latitude $\Phi=65^{\circ}$. The station records with a 1 min sampling rate a quasiDC current in the dead-grounded neutral of a transformer. We analyze the Pc5/Pi3s pulsations detected during the year of 2015 .

The Kevo (KEV) magnetic station is taken as the basic one, as it lies at the shortest distance from VKH and nearly at the same geomagnetic latitude. The Sodankylä (SOD) and Kilpisjärvi (KIL) stations are used for estimates of the pulsation's meridional and latitudinal spatial scales, respectively. Station locations are shown on the map (Figure 1) and the station information is summarized in Table 1.

The following technique is used for the analysis of the pulsations. Both the geomagnetic and GIC data are high-pass filtered with a $0.8 \mathrm{mHz}$ cutoff frequency and decimated to a common 1-minute sampling rate. Then, the spectral estimates are made in a 64 point $(3840 \mathrm{~s})$ running window with a 5 -min shift between subsequent intervals for the GIC and the two horizontal components of the magnetic field. The Periodic ULF disturbances are automatically selected with a detection program for the time intervals with a pronounced spectral maximum over the background "colored noise" spectrum (Yagova et al, 2015). The results of this selection have been visually checked. The bandwidth analyzed comprises the Pi3 range (predominantly $f<2 \mathrm{mHz})$ and $\mathrm{Pc} 5$ range $(f>1.7 \mathrm{mHz})$.

The efficiency of the GIC generation is taken into account with a $R_{I-B}$ parameter, i.e. the PSD ratio of GIC to the magnetic field component at a given frequency. For each pulsation interval, $R_{I-B}$ is calculated and its dependence on pulsation frequency and spatial scale is analyzed. Horizontal inhomogeneity of both magnetic field and crust electric properties leads to an essential contribution to the GIC generation of both horizontal components of the geomagnetic field. Besides, the parameters of the two components are closely inter-related. However, the influence of $B_{Y}$ component on GIC in a meridional electric power line is stronger, and we use for statistical purposes.

Both the absolute values and angles between the wave amplitude and phase gradients and the EPL are important for GIC generation. First, we look at the GIC efficiency of Pc5/Pi3s in dependence on the pulsation scale transversal to the EPL. The power line is oriented along the magnetic meridian. Thus, we shall analyze the E-W scale. As it was shown in Yagova et al (2018), the Pi3 pulsations can be localized in the E-W direction and prolongated in the N-S direction. This leads to interpolation/extrapolation errors. For this problem, parameters, averaged over relatively long time intervals, depend mostly on pulsations' amplitude (spectral power) ratio and spectral content (resulting in spectral coherence), while the phase difference contribution is expected to be small.

As for the pulsation scale parallel to an EPL, it influences the magnetic flux variation in the contour, if the amplitude and/or phase of pulsation changes essentially at the power line length. Finally, for this direction, GIC is determined by the shorter of the two scales: the EPL length and pulsation's scale. 
In the present study, we use the same classification of the large- and small-scale pulsations, as in Sakharov et al (2020). For the meridional/latitudinal directions we use the KEV-SOD and KEV-KIL station pairs, separated from KEV by $\Delta \Phi=2.4^{\circ}$ $(270 \mathrm{~km})$ and $\Delta \Lambda=5.5^{\circ}(250 \mathrm{~km})$, respectively. For the E-W direction, we define the pulsation at a frequency $f$ as a large-scale $\left(L_{E W}\right)$, if spectral coherence at this frequency is high and the PSD ratio is close to 1.

A hypothesis to check is that the $L_{E W}$ pulsations demonstrate higher spectral coherences with GIC variations than the small-scale $S_{E W}$ ones.

For the N-S (parallel to the power line) direction, also a phase difference becomes important, because only in the case of a small phase difference at the EPL length, an essential part of the EPL length will contribute to the Electromotive force (EMF). The second factor is the amplitude dependence on the coordinate (latitude in our case). As the station pair used in the present study is located at the North of the EPL latitude interval, the pulsation efficiency should be higher for the pulsations with a higher South-to-North PSD ratio $R_{S N, B y}$. That is, for the N-S direction, we define a pulsation as large-scale $\left(L_{N S}\right)$, if it is characterized by three parameters: a high spectral coherence, low phase difference, and high South-to-North PSD ratio.

A hypothesis to check is that the $L_{N S}$ pulsations generate more intensive GICs than the small-scale $\left(S_{N S}\right)$ ones of the same amplitude and frequency, i.e. that the $R_{I-B y}$ is higher for the $L_{N S}$ than for the $S_{N S}$ pulsations.

The boundary values for coherence, phase difference and South-to-North PSD ratio are equal to $\gamma_{b}=0.7, \mu_{b}=0.85(\mu=\cos (\Delta \varphi))$, and $R_{S N, B y}=0.5$, respectively. These values are selected in such a way that each resulting group has a comparable number of events.

\section{Results}

Examples of Pc5s with different GIC efficiency

A large-scale pulsation registered on 1 March 2015 (day 60) at 7:15 UT

Waveforms and spectral parameters of Pc5 pulsations, recorded simultaneously in the geomagnetic field at KEV and in the GIC at VKH at local morning (10.5 MLT at KEV), are given in Figure 2. The pulsation's main period is approximately 4 minutes. The peak-to-peak amplitude of the pulsation varies from 20 to $40 \mathrm{nT}$ for the geomagnetic field and 5-10 A for the GIC (Figure 2a). The PSD spectra of both the magnetic field and the GIC demonstrate maxima at $f_{1}=1.7, f_{2}=2.7$, and $f_{3}=4 \mathrm{mHz}$ (Figure 2b), and the spectral coherence $\gamma_{I-B y}^{2}$ (Figure 2c) is almost 1 at all the frequency band $1.7-5 \mathrm{mHz}$. This panel also shows the spectral coherence $\gamma_{I-B x}^{2}$ between the $B_{X}$ component and the GIC. Although it exceeds 0.5, it is lower than $\gamma_{I-B y}^{2}$ at all the frequencies analyzed. $R_{I-B y}$ varies in the range of $0.01-0.03 \mathrm{~A}^{2} / \mathrm{nT}^{2}$ and it has a maximum at the $f_{2}$ frequency and it grows with frequency at $f>3.3 \mathrm{mHz}$ (Figure $2 \mathrm{~d}$ ).

What about the spatial properties of this pulsation? The pulsation properties in the E-W direction are illustrated by Figure 3. For the KEV-KIL station pair located along the geomagnetic latitude $\Phi=66^{\circ}$ with a $5.5^{\circ}$ shift in longitude, the pulsation's waveforms are similar (Figure 3a). This is also confirmed by spectral parameters (Figure 3, b-d). The spectral coherence $\gamma_{I-B y}^{2}$ exceeds 0.5 (Figure 3c) for the frequencies of all the spectral maxima found in the KEV PSD spectrum. The 
spectral coherence $\gamma_{I-B x}^{2}$ is almost 1 . The East-to-West PSD spectral ratio $R_{E W, B y}$ at these frequencies exceeds 1 . The $R_{E W, B x}$ ratio is even higher, than $R_{E W, B y}$, and it exceeds 1 at all frequencies analyzed (Figure $3 \mathrm{~d}$ ).

Thus, we classify this as a large-scale pulsation in the E-W direction. The found spectral ratio and coherence allow us to suggest that at the $\Lambda=113^{\circ}$, i.e. at the EPL longitude, the pulsation should have nearly the same spectral content, as at KEV, and a comparable (probably, somewhat higher) amplitude.

The pulsation properties in the N-S direction are illustrated in Figure 4. Waveforms for both horizontal components are shown on the left of the Figure (4a,b). The pulsation in the $B_{Y}$ component is clearly seen at SOD, but its amplitude is lower, than at KEV. The spectral coherence is almost 1 for all the three spectral maxima. At the $f_{1}$ frequency, the pulsations are in phase. At the two higher frequencies, the phase difference does not exceed $25^{\circ}$. Thus, the sign of $d B_{Y} / d t$ remains the same during almost all of the pulsation half-period. The South-to-North PSD ratio changes from 1 at $1.7 \mathrm{mHz}$ to about 0.3 (i.e. the amplitude ratio is about $0.55)$ at the frequencies of the other two spectral maxima. The variations of $B_{X}$ component are almost counter-phased to $B_{Y}$, and the spectral maximum at $f_{2}$ is higher than that in $B_{Y}$ and in $B_{X}$ at all the other frequencies. The absolute value of phase difference in $B_{X}$ does not exceed $45^{\circ}$ at $f<4 \mathrm{mHz}$, and its sign at $f_{2}$ is positive, in contrast to that in $B_{Y}$. The South-to-North PSD ratio in $B_{X}$ is about 0.3 at these frequencies.

This pulsation is polarized almost linearly and it demonstrates high coherence and low phase difference in both horizontal components. The difference between the two components in the PSD and phase spectra near $f_{2}$ can result from the FLR resonance, which is expressed predominantly in $B_{X}$. Thus, we classify this as a large-scale pulsation in the N-S direction. We expect that it should be effective for GIC generation. Actually, GIC to BY PSD ratio varies with frequency in the $0.01-0.03 \mathrm{~A}^{2} / \mathrm{nT}^{2}$ range (i.e., $0.1-0.2 \mathrm{~A} / \mathrm{nT}$ in the amplitude spectra).

A small-scale pulsation registered on 12 May 2015 (day 132) at 4:05 UT

The pulsation recorded in the early morning (7 MLT at KEV) of May 12 is illustrated in Figure 5. The peak-to peak amplitude of the geomagnetic pulsation at KEV reaches $60 \mathrm{nT}$. Simultaneously, the pulsation is seen in GIC with the amplitude of about $1 \mathrm{~A}$ (Figure 5a). The main spectral maximum is found in the PSD spectra at $f_{1}=2.1 \mathrm{mHz}$, and a minor maximum - at $f_{2}=3.7 \mathrm{mHz}$ (Figure $5 \mathrm{~b}$ ). Both frequencies are stressed in the coherence spectrum, as well. The coherence between GIC and $B_{X}$ is lower than that between GIC and $B_{Y}$ (Figure $5 \mathrm{c}$ ). It should also be noted, that the spectral coherence in all the frequency range is lower than in the previous event. The GIC to $B_{Y}$ PSD ratio varies near $R_{I-B y}=3 \cdot 10^{-4} \mathrm{~A}^{2} / \mathrm{nT}^{2}$ (Figure 5d), i.e. it is two orders of magnitude lower, than for the previous event.

The pulsation waveforms at the KEV-KIL station pair and their spectral parameters are presented in Figure 6 . The $B_{Y}$ pulsation is seen at both stations with similar waveforms and comparable amplitudes (Figure 6a). Both frequencies of spectral maxima at KEV, can also be seen in the KIL PSD spectrum (Figure 6b). However, a maximum in the coherence spectrum is only found for the $f_{1}$ frequency with $\gamma^{2}=0.9$, while at $f_{2}, \gamma^{2}$ is about 0.5 . The $B_{X}$ coherence spectrum is similar to 
that of $B_{Y}$ at $f<2.4 \mathrm{mHz}$ and then $\gamma_{E W, B x}^{2}$ decreases with $f$ quicker than $\gamma_{E W, B y}^{2}$. For the $B_{X}$ component, the East-to-West PSD ratio $R_{E W, B x}$ exceeds 1 at all the frequencies, while for $B_{Y}$, it is nearly 1 at $f_{1}$ and about 0.3 at $f_{2}$. This allows us to assume that the pulsation should be seen in $B_{Y}$ at the VKH longitude with an amplitude close to that at KEV at $f_{1}$ and with a somewhat lower amplitude at $f_{2}$.

The meridional variations of the pulsation parameters are shown in Figure 7 . In both components, the pulsation is seen at the KEV and SOD stations with similar apparent periods, but its amplitude and phase differ essentially (note, that we use the different vertical scale for the two stations to make the pulsation at SOD visible). As a result, the sign of $d B_{Y} / d t$ remains the same between KEV and SOD only during an approximately quarter of the pulsation period (the time is twice shorter, than in the previous case). The spectral peak at the $f_{1}$ frequency is seen in both the PSD and coherence spectra (Figure 7, bc). As for the South-to-North PSD ratio, it is about 0.03 ( 0.2 in the amplitude spectra). The $B_{X}$ coherence is lower, the phase difference is nearly the same, and the South-to-North spectral ratio $\left(R_{S N, B x}\right)$ is higher than the corresponding parameters for the $B_{Y}$ component. According to the selection criteria, this pulsation is small-scale in both directions.

The comparison of pulsation amplitudes in GIC and geomagnetic components for the two events analyzed demonstrates that the first pulsation is more effective in GIC generation than the second one. In fact, the GIC amplitude during the second interval is only about $1 \mathrm{~A}$, i.e. it is an order of magnitude lower than for the first event, while the amplitude of the geomagnetic pulsation is higher in the second case. We assume that this results from the difference of spatial scales of the pulsations. In the next subsection we shall verify this assumption using the analysis of pulsations registered in $B_{Y}$ component of the geomagnetic field and GIC during the year 2015 .

\section{Statistics}

The influence of the pulsation scale in the $\mathrm{E}-\mathrm{W}$ direction (i.e. transversal to the EPL) is more "technical". It can cause problems with data interpolation (or extrapolation), when the magnetic field and GIC are measured at different locations. Spectral coherence $\gamma_{I-B y}^{2}$ quantifies the inter-dependence between GIC and magnetic field variations. The normalized probability density functions (PDFs) over $\gamma_{I-B y}^{2}$ are given in Figure 8 for the large-scale $\left(L_{E W}\right)$ and small-scale $\left(S_{E W}\right)$ pulsations. The difference in distributions is clearly seen in all the frequency bands. A fraction of low-coherent intervals is essentially higher for the small-scale pulsations, while the large-scale pulsations demonstrate a pronounced high-coherence maxima at all the frequencies.

On the contrary, the spatial scale in the N-S direction (parallel to the EPL), influences the GIC generation directly. Large-scale pulsations generate GICs with the amplitudes higher, than small-scale pulsations of the same amplitude. This effect is seen in distribution of the two groups of pulsations over the PSD ratio $R_{I-B y}$. Normalized PDFs over $R_{I-B y}$ for the same frequency bands, as in the previous Figure, are shown in Figure 9 for the large-scale $\left(L_{N S}\right)$ and small-scale $\left(S_{N S}\right)$ pulsations. For all the frequency bands, the distributions for the small-scale pulsations are enriched with low values of $R_{I-B y}$. At the two lower frequencies, the most probable value of $R_{I-B y}$ is the same for the two groups of pulsations, while for 
the two higher frequencies, the most probable $R_{I-B y}$ is also higher for the large-scale pulsations. The fraction of $R_{I-B y}>0.1 \mathrm{~A}^{2} / \mathrm{nT}^{2}(0.3 \mathrm{~A} / \mathrm{nT}$ in amplitude spectra) is nearly two times higher for the large-scale pulsations, than for the small-scale ones in all the frequency bands. As for the rare events $\left(F^{*} \simeq 10^{-3}\right)$ with extremely high values of $R_{I-B y} \geq 1 \mathrm{~A}^{2} / \mathrm{nT}^{2}$, their fraction is even higher for the small-scale pulsations, than for the large-scale ones. This effect should be a point of a special study.

Actually, we have used three parameters to discriminate between large-scale and small-scale pulsations, namely, the spectral coherence, phase difference, and Southto-North PSD ratio. In a real wave, they are not independent. However, we can try to discriminate between their influence on $R_{I-B y}$. A low coherence at a given frequency means that the phase difference changes essentially during the time interval, for which the spectrum is calculated. Thus, phase difference estimates are valid only for coherent pulsations. We expect that the coherence and phase difference influence $R_{I-B y}$ in a similar way, because both the low coherence and high phase difference at the EPL length lead to a situation, where different EPL segments contribute to the EPF with the opposite signs. On the contrary, the South-to-north PSD ratio RSN,By, influences the GIC only via EPF amplitude variation along the contour. Thus, the $R_{I-B y}$ dependence on the $R_{S N, B y}$ is expected to be weaker than its dependence on coherence and phase difference.

The result of this analysis in the form of the averaged $R_{I-B y}$ spectrum for several groups of pulsations is given in Figure 10. First, we divide pulsations into smallscale and large-scale ones, depending on their spectral coherence (marked $S_{\gamma}$ and $L_{\gamma}$ in the Figure). Then, we divide the $L_{\gamma}$ group into small-scale and large-scale subgroups in accordance to their phase difference $\left(L_{\gamma} S_{\varphi}\right.$ and $L_{\gamma} L_{\varphi}$ in the Figure). At the last stage, we divide the $L_{\gamma} L_{\varphi}$ group into small-scale and large-scale sub-groups depending on their South-to-North PSD ratio RSN,By $\left(L_{\gamma} L_{\varphi} S_{P}\right.$ and $L_{\gamma} L_{\varphi} L_{P}$ in the Figure). The spectral coherence and phase difference influence the $R_{I-B y}$ spectra to more or less the same degree (the boundaries taken at the levels $\gamma_{b}=0.7, \mu_{b}=0.85$, where $\mu=\cos (\Delta \varphi)$, and $\left.R_{S N, B y}=0.5\right)$.

In contrast to the other groups, the spectrum of the coherent one with a high phase difference $L_{\gamma} S_{\varphi}$ is not monotonous, and it has a maximum at $f=3.3 \mathrm{mHz}$. The average value of $R_{I-B y}$ ratio is nearly three times higher for $L_{\gamma}$ and $L_{\gamma} L_{\varphi}$ groups than for the $S_{\gamma}$ and $L_{\gamma} S_{\varphi}$ ones. The $R_{I-B y}$ ratio for pulsations defined as large-scale using all three parameters $\left(L_{\gamma} L_{\varphi} L_{P}\right.$ group) is about 2 times higher than that for the $L_{\gamma} L_{\varphi} S_{P}$ group. This demonstrates that the $R_{I-B y}$ dependence on PSD variation along the profile is weaker than its dependence on phase variation. As for the $R_{I-B y}$ ratio for the most effective $L_{\gamma} L_{\varphi} L_{P}$ group, it is 4 times higher than that for the $S_{\gamma}$ group group, for which the GIC efficiency is minimal.

In the final analysis, we return to the classification of pulsations into two groups and define the $L_{\gamma} L_{\varphi} L_{P}$ pulsations only, as large-scale $\left(L_{N S}\right)$. All the other pulsations are considered to be small-scale $\left(S_{N S}\right)$. The resulting averaged $R_{I-B y}$ spectra for these two groups are given in Figure 11(a). The large-scale pulsations produce a higher average PSD in GIC than the small-scale ones. The $R_{I-B y}$ ratio grows from $1.5 \cdot 10^{-2} \mathrm{~A}^{2} / \mathrm{nT}^{2}$ at $1.5 \mathrm{mHz}$ to $4.4 \cdot 10^{-2} \mathrm{~A}^{2} / \mathrm{nT}^{2}$ at $5 \mathrm{mHz}$ for the largescale pulsations and from $5 \cdot 10^{-3}$ to $2.2 \cdot 10^{-2} \mathrm{~A}^{2} / \mathrm{nT}^{2}$ for the small-scale ones. 
Its averaged over the frequency band value is three times higher for the large-scale pulsations than for the small-scale ones.

The pulsations' efficiency in GIC production is characterized not only by the mean values of GIC amplitudes, but also by a fraction of high $R_{I-B y}$ values. At the lower panel of the Figure $(11, \mathrm{~b})$, the frequency dependence of $R_{I-B y}>0.1 \mathrm{~A}^{2} / \mathrm{nT}^{2}$ probability $P_{0.1}$ is shown for the same two groups of pulsations, as at the 11a panel. $P_{0.1}$ is 2 to 3 times higher for the large-scale than for small-scale pulsations, and at $f>3 \mathrm{mHz}$ it exceeds 0.1, i.e. at these frequencies for each tenth interval the GIC to By PSD ratio exceeds $0.1 \mathrm{~A}^{2} / \mathrm{nT}^{2}$.

\section{Discussion}

Using the GIC and magnetic field data recorded in Russian North and Fennoscandia in 2015, we have analyzed the influence of Pc5/Pi3 spatial scale on the efficiency of GIC generation. Our results are based on the analysis of the $B_{Y}$ component of geomagnetic pulsations. Meanwhile, the horizontal inhomogeneity of crust electric properties and the high coherence between the two components lead to the essential GIC dependence on parameters of both horizontal components. The telluric electric field in realistic geoelectric conditions is more inhomogeneous in amplitude and direction than the primary magnetic field variations. Bedrosian and Love (2015) demonstrated that a telluric E-field can exhibit rapid spatial variations related to the variations in the electrical conductivity of the Earth, even in the presence of a spatially uniform B-field. Therefore, the role of both magnetic components should be examined with the special technique used for discrimination between inter-dependent parameters.

The GIC to $B_{Y}$ PSD spectral ratio $R_{I-B y}$ varies from $10^{-4}$ to $1 \mathrm{~A}^{2} / \mathrm{nT}^{2}$ with most probable values of $1-3 \cdot 10^{-2} \mathrm{~A}^{2} / \mathrm{nT}^{2}$ depending on pulsation frequency and spatial scale. The pulsation scale in the E-W direction (transversal to the EPL), is important, because the errors of extrapolation and interpolation are higher for small-scale pulsations. For more accurate estimates of GIC amplitudes, more dense networks of magnetometers are necessary.

As for the N-S (parallel to the EPL direction) distribution of the pulsation amplitude and phase, it influences the GIC amplitude directly. The large-scale pulsations (i.e. those with a high coherence, low phase difference, and higher South-to-North spectral ratio) produce more intensive GICs than the short-scale pulsations of the same amplitudes. The comparison of the three parameters, used for pulsations classification into large- and small-scale ones, has shown that the parameters accumulating phase information, i.e. coherence and phase difference, demonstrate stronger influence on the GICs than the PSD meridional distribution.

A non-monotonous dependence of $R_{I-B y}$ on frequency, found for the group of coherent pulsations with the high phase difference, is probably associated with the Alfven resonance effect (Baransky et al, 1995). Although, this effect is expressed brightly in $B_{X}$, weaker resonance features are also found in the meridional distribution of the $B_{Y}$ amplitude and phase (?). In the vicinity of a resonance latitude, phase and amplitude gradients are higher than at non-resonant latitudes and the pulsation amplitude grows southward. In the case of a meridional EPL, $R_{I-B y}$ decreases with the absolute value of phase difference. Meanwhile, for the system 
analyzed, $R_{I-B y}$ increases with the South-to-North PSD ratio. As a result, the phase and amplitude gradients have opposite influence on the efficiency of the GIC generation. The combination of these two factors can lead to a non-monotonous dependence of $R_{I-B y}$ on frequency.

The statistical analysis of pulsation intervals recorded during the year 2015 has shown that the yearly mean values of $R_{I-B y}$ are about three times higher for the N-S large-scale $\left(L_{N S}\right)$ pulsations, than for the small-scale $\left(S_{N S}\right)$ ones. Meanwhile, the integral over $1.5-5 \mathrm{mHz}$ band values of $R_{I-B y}$, calculated within uniform conductivity and the constant contour depth models, differ by no more than two times. This means, that the pulsations' spatial scale role in the GIC generation may be even more important, than the details of crust conductivity distribution with depth. Although the Pc5 properties are studied in detail, the knowledge of their spatial distribution is not enough for GIC application, especially for $B_{Y}$ component. It is even more so for irregular pulsations, like Pi3s, Ps6, etc.

The slopes of the $R_{I-B y}$ spectra, shown in Figure 11, differ for the two groups of pulsations. For the large-scale pulsations, it corresponds to a model of constant crust conductivity ( $f^{1 / 2}$ in amplitude spectra), while for the small-scale ones, it is very close to the linear dependence on frequency, i.e. to a model with a constant contour depth. Under the average conductivity in Fennoscandia of about $10^{-4} \mathrm{~S} / \mathrm{m}$, the skin depth for the $1.5-5 \mathrm{mHz}$ frequency band is about several hundred kilometers, i.e. the wavelength of the large-scale pulsations exceeds the skin depth and the model of constant conductivity can be used. In the case of small-scale pulsations, the maximal contour depth is determined by wavelength, and, thus, the model of a constant contour depth can be used.

\section{Conclusion}

The pulsation spatial scale in the frequency range of several milliHertz (Pc5/Pi3) influences the parameters that describe the inter-relation between geomagnetic and GIC pulsations. The statistical and case studies of spatial scale of BY component of Pc5/Pi3 pulsations, recorded at auroral latitudes in Fenno-Scandia in 2015, have shown that the scale of a pulsation in the E-W direction (transversal to the EPL), increases the coherence between geomagnetic and GIC variations in the $\mathrm{Pc5} / \mathrm{Pi} 3$ frequency range. The large-scale in the N-S direction (parallel to the EPL) pulsations generate more intensive geoelectric currents, than the small-scale pulsations of the same amplitudes. For the large-scale pulsations, this results in mean the GIC to $B_{Y}$ PSD ratio $R_{I-B y}$ about three times higher, than for the small-scale pulsations. The yearly mean value of $R_{I-B y}$ varies from $1.5 \cdot 10^{-2} \mathrm{~A}^{2} / \mathrm{nT}^{2}$ at $1.5 \mathrm{mHz}$ to $4.4 \cdot 10^{-2} \mathrm{~A}^{2} / \mathrm{nT}^{2}$ at $5 \mathrm{mHz}$ for the large-scale pulsations and from $5 \cdot 10^{-3}$ to $2.2 \cdot 10^{-2} \mathrm{~A}^{2} / \mathrm{nT}^{2}$ for the small-scale pulsations. A higher efficiency of long-scale pulsations is also seen in the probability to register high $R_{I-B y}$ values: the fraction of $R_{I-B y}>0.1 \mathrm{~A}^{2} / \mathrm{nT}^{2}$ is about two times higher for the large-scale pulsations than for the small-scale ones.

Availability of data and materials

Calculated spectra are available as supplementary files.

Competing interests

The authors declare that they have no competing interests. 
Author's contributions

N.V. Yagova: data processing and interpretation of results; V.A. Pilipenko: interpretation of results, review and analysis of previous studies, theoretical estimates; Ya.A. Sakharov: GIC data preliminary analysis and selection of events; V.N. Selivanov: providing of GIC obsevration, preliminary data processing; all the authors: preparation of the MS.

\section{Funding}

This work was supported by the Russian Science Foundation, Grant 16-17-00121.

Acknowledgements

We thank the institutes who maintain the IMAGE Magnetometer Array: Troms $\varnothing$ Geophysical Observatory of UiT the Arctic University of Norway (Norway), Finnish Meteorological Institute (Finland), Institute of Geophysics Polish Academy of Sciences (Poland), GFZ German Research Centre for Geosciences (Germany), Geological Survey of Sweden (Sweden), Swedish Institute of Space Physics (Sweden), Sodankylä Geophysical Observatory of the University of Oulu (Finland), and Polar Geophysical Institute (Russia).

Author details

${ }^{1}$ Schmidt Institute of Physics of the Earth of the Russian Academy of Sciences (IPE RAS), B. Gruzinskaya ul, 10, Moscow, Russia. ${ }^{2}$ The Geophysical Center of the Russian Academy of Sciences (GC RAS), Molodezhnaya ul., 3, Moscow, Russia. ${ }^{3}$ Polar Geophysical Institute, Akademgorodok, 26A, Apatity, Russia. ${ }^{4}$ Northern Energetics Research Center, Kola Science Center of the Russian Academy of Sciences Akademgorodok, 21A, Apatity, Russia.

\section{References}

Apatenkov SV, Sergeev VA, Pirjola R, Viljanen A (2004) Evaluation of the geometry of ionospheric current systems related to rapid geomagnetic variations. Ann Geophys 22:63-72

Apatenkov SV, Pilipenko VA, Gordeev EI, Viljanen A, Juusola L, Belakhovsky VB, Sakharov YA, N SV (2020) Auroral omega bands are a significant cause of large geomagnetically induced currents. Geophys Res Lett 47(e2019GL086677)

Baransky LN, Fedorov EN, Kurneva NA, Pilipenko VA, Green AW, Worthington EW (1995) Gradient and polarization methods of the ground-based hydromagnetic monitoring of magnetospheric plasma. J Geomagn Geolect 47:1293-1309

Bedrosian PA, Love JJ (2015) Mapping geoelectric fields during magnetic storms: Synthetic analysis of empirical united states impedances. Geophys Res Lett 42:10,160-10,170,

Belakhovsky V, Pilipenko V, Sakharov Y, V S (2018) Characteristics of the variability of a geomagnetic field for studying the impact of the magnetic storms and substorms on electrical energy systems. Izvestiya, Physics of the Solid Earth 54(1)

Belakhovsky V, Pilipenko V, Engebretson M, Sakharov Y, V S (2019) Impulsive disturbances of the geomagnetic field as a cause of induced currents of electric power lines. Journ Space Weath Space Clim 9(A18)

Boteler DH (2001) Space weather effects on power systems. In: Song SHJ P, Siscoe G (eds) Space Weather, AGU, Washington, pp 347-352

Boteler DH, Pirjola RJ (2017) Modeling geomagnetically induced currents. Space Weath 15:258- 276

Engebretson MJ, Steinmetz ES, Posch JL, Pilipenko VA, Moldwin MB, Connors MG, Boteler DH, Mann IR, Hartinger MD, Weygand JM, Lyons LR, Nishimura Y, Singer HJ, Ohtani S, Russell CT, Fazakerley SA, A KS (2019) Nighttime magnetic perturbation events observed in arctic canada: 2. multiple-instrument observations Journ Geophys Res: Space Phys 124,

Kappenman J (2004) The evolving vulnerability of electric power grids. Space Weather 2(S01004)

Love JJ, Lucas GM, Kelbert A, Bedrosian PA (2018) Geomagnetically induced currents modeling and forecasting. Space Weather 16:1114-1127

Pulkkinen A (2015) Geomagnetically induced currents modeling and forecasting. Space Weather 13:734-736

Pulkkinen A, Kataoka R (2006) S-transform view of geomagnetically induced currents during geomagnetic superstorms. Geophys Res Lett 33(L12108)

Pulkkinen A, Hesse M, Kuznetsova M, Rastssatter L (2007) First-principles modeling of geomagnetically induced electromagnetic fields and currents from upstream solar wind to the surface of the earth. Ann Geophys 25:881-893

Sakharov YA, Yagova NV, Pilipenko VA (2020) Pc5/pi3 geomagnetic pulsations and geomagnetically induced currents. In press

Tanskanen El (2009) A comprehensive high-throughput analysis of substorms observed by image magnetometer network: Years 1993-2003 examined. J Geophys Res 114(A05204):1-1,

Viljanen A (1998) Relation of geomagnetically induced currents and local geomagnetic field variations. Trans Power Deliv 13:1285-1290

Viljanen A, Nevanlinna H, Pajunpaa K, Pulkkinen A (2001) Time derivative of the horizontal geomagnetic field as an activity indicator. Ann Geophys 19(A17):1-1,

Viljanen A, Pirjola R, Wik M, Adam A, Pracser E, Sakharov Y, Katkalov J (2012) Continental scale modelling of geomagnetically induced currents. J Space Weather Space Clim 2:1107-1118

Yagova NV, Heilig B, Fedorov EN (2015) Pc2-3 geomagnetic pulsations on the ground, in the ionosphere, and in the magnetosphere: Mm100, champ, and themis observations. Ann Geophys 33:117-128

Yagova NV, Pilipenko VA, Fedorov EN, Lhamdondog AD, Gusev YP (2018) Geomagnetically induced currents and space weather: $\mathrm{Pi} 3$ pulsations and extreme values of time derivatives of the geomagnetic field's horizontal components. Izvestiya, Physics of the Solid Earth 54:749-763

Figures

Tables 
Figure 1 Locations of the magnetic observatories, the observational point of GIC recording at $\mathrm{VKH}$. The electric power line is schematically shown with a red dashed line

Figure 2 Event 1 Pulsations recorded in the geomagnetic field and GIC on day 2015060 . (a) waveforms of $B_{Y}$ pulsations at KEV (magenta) and GIC at VKH (dark blue); (b) normalized PSD spectra; (c) $B-I$ spectral coherence for $B_{Y}$ (solid line) and $B_{X}$ - (dashed); (d) GIC to $B_{Y}$ PSD ratio

Figure 3 Parameters of the event 1 pulsation in the East-West (E-W) direction: (a) $B_{Y}$ waveforms at KEV (magenta) and KIL (green); (b) normalized PSD spectra; (c) E-W spectral coherence for $B_{Y}$ (solid) and $B_{X}$ (dashed); (d) E-W PSD ratio for $B_{Y}$ (solid), and $B_{X}$ (dashed)

Figure 4 Parameters of the event 1 pulsation in the North-South (N-S) direction: (a) and (b) $B_{Y}$ and $B_{X}$ waveforms at KEV (magenta) and SOD (blue); (c) normalized PSD spectra; (d) and (e) NS spectral coherence and phase difference for $B_{Y}$ (solid) and $B_{X}$ - (dashed); (f) South-to-North PSD ratio for $B_{Y}$ (solid) and $B_{X}$ - (dashed)

Figure 5 Event 2 Pulsations recorded in the geomagnetic field and GIC on day 2015 132. (a) waveforms of $B_{Y}$ pulsations at KEV (magenta) and GIC at VKH (dark blue); (b) normalized PSD spectra; (c) $B-I$ spectral coherence for $B_{Y}$ (solid) and $B_{X}$ (dashed); (d) $I$ to $B_{Y}$ PSD ratio

Figure 6 Parameters of the event 2 pulsation in the East-West (E-W) direction: (a) $B_{Y}$ waveforms at KEV (magenta) and KIL (green); (b) normalized PSD spectra; (c) E-W spectral coherence for $B_{Y}$ (solid) and $B_{X}$ (dashed); (d) E-W PSD ratio for $B_{Y}$ (solid) and $B_{X}$ (dashed)

Figure 7 Parameters of the event 2 pulsation in the North-South (N-S) direction: (a) and (b) $B_{Y}$ and $B_{X}$ waveforms at KEV (magenta) and SOD (blue); (c) normalized PSD spectra; (d) and (e) N-S spectral coherence and phase difference for $B_{Y}$ (solid) and $B_{X}-$ (dashed); (f) South-to-North PSD ratio for $B_{Y}$ (solid) and $B_{X}$ - (dashed)

Figure 8 Empirical probability density over $I-B_{Y}$ spectral coherence $\gamma_{I-B y}^{2}$ for the pulsations of two groups: large-scale $\left(L_{E W}\right)$ and small-scale $\left(S_{E W}\right)$ in the East-West direction

Figure 9 Empirical probability density over $I-B_{Y}$ PSD ratio $R_{I-B y}$ for the pulsations large-scale $\left(L_{N S}\right)$ and small-scale $\left(S_{N S}\right)$ in the North-South direction

Figure 10 Averaged $R_{I-B y}$ spectra for 6 groups of pulsations: 1)-2) small-scale $S_{\gamma}$ and large-scale $L_{\gamma}$ in accordance to the N-S spectral coherence; 3)-4) $L_{\gamma} S_{\varphi}$ and $L_{\gamma} L_{\varphi}$ are the small-/large-scale sub-groups of the $L_{\gamma}$ group defined in accordance to the phase difference; 5)-6) $L_{\gamma} L_{\varphi} S_{P}$ and $L_{\gamma} L_{\varphi} L_{P}$ are the small- /large-scale sub-groups of the $L_{\gamma} L_{\varphi}$ groups defined in accordance to the South-to-North PSD ratio.

Figure 11 (a) Averaged $R_{I-B y}$ spectra for the pulsations large-scale $\left(L_{N S}\right)$ and small-scale $\left(S_{N S}\right)$ in the North-South direction. ( $L_{N S}=L_{\gamma} L_{\varphi} L_{P}$ in Figure 10, while all the other groups form the $S_{N S}$ group) ; (b) frequency dependence of $R_{I-B y}>0.1 \mathrm{~A}^{2} / \mathrm{nT}^{2}$ fraction for the same groups of pulsations 
Table 1 Stations Information

\begin{tabular}{|c|c|c|c|c|c|c|}
\hline Station & Code & $\begin{array}{l}\text { Geographic } \\
\text { LAT }\end{array}$ & LON & $\begin{array}{l}\text { CGM } \\
\text { LAT }(\Phi)\end{array}$ & $\operatorname{LON}(\Lambda)$ & $\begin{array}{l}\text { UT of MLT } \\
\text { midnight }\end{array}$ \\
\hline Kevo & KEV & 69.76 & \begin{tabular}{|l|}
27.01 \\
\end{tabular} & 66.65 & 108.35 & $21: 06$ \\
\hline Kilpisjärvi & KIL & 69.02 & 20.79 & 66.13 & 102.80 & $21: 28$ \\
\hline Sodankylä & SOD & 67.37 & 26.63 & 64.22 & 106.52 & $21: 13$ \\
\hline Vykhodnoy & VKH & 68.83 & 33.08 & 65.53 & 112.73 & $20: 49$ \\
\hline
\end{tabular}

